\title{
III. Note on the application of Cornu's spiral to the diffraction-grating.-A geometrical method of obtaining the intensity formula for a flat diffraction-grating
}

\section{Arthur L. Kimball Ph.D.}

To cite this article: Arthur L. Kimball Ph.D. (1903) III. Note on the application of Cornu's spiral to the diffraction-grating.-A geometrical method of obtaining the intensity formula for a flat diffraction-grating , Philosophical Magazine Series 6, 6:31, 30-33, DOI: 10.1080/14786440309462984

To link to this article: http://dx.doi.org/10.1080/14786440309462984

$$
\text { 曲 Published online: } 15 \text { Apr } 2009 .
$$

Submit your article to this journal ए

\section{Џ Article views: 3}

Q View related articles 5 


\section{$\left[\begin{array}{ll}30 & ]\end{array}\right.$}

III. Note on the Application of Cornu's Spiral to the Diffraction-Grating.-A Geometrical Method of obtaining the Intensity Formula for a Flat Difraction-Grating. By Arthur L. Kimball, Ph.D., Professor of Physics in Amkerst College".

T $\mathrm{T}$ is well known that the graphic method of Cornu furnishes a useful method of discussing many diffraction phenomena, notably the case of a single narrow slit; but, so far as I am aware, the formula for the intensity of the light diffracted by a grating of $n$ lines has not been obtained without involving the summation by analytic process of the trigonometric series $\sin x+\sin (x+y)+\sin (x+2 y)+\& c \ldots \sin (x+(n-1) y)$.

In the following note it is shown how, by a simple geometrical device, the result may be obtained at a stroke; and the method besides giving immediately the desired summation makes the discussion of the result particularly direct and simple. When a flat wave falls on a flat diffraction-grating made up of alternate bars and spaces, the graphic expression of the amplitudes and phases of vibration due to the waves through the several slits, is a series of chords of Cornu's spiral.

In case the resultant amplitude is sought at a point at an infinite distance from the grating, or for a point in the focal plane of the observing telescope, Cornu's spiral becomes a circle.

Thus the chord $c d$ will represent the amplitude and phase of motion due to the first slit, of that due to the second, $g h$

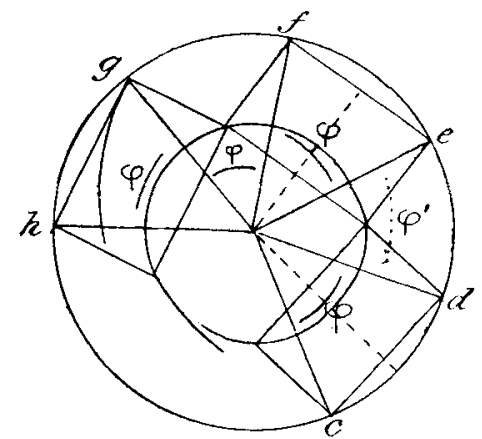

that of the third, \&c., and if the slits are all equal and equally far apart, the amplitudes will be a series of equal chords at equal distances apart around the circle, and the resultant amplitude is the geometrical sum of these chords.

* Communicated by the Author. 
Application of Cornu's Spiral to Diffraction-Grating.

Suppose the incident wave-front is parallel with the grating, and that the direction of the point $\mathrm{P}$, where the resultant amplitude is sought, makes an angle $\theta$ with the normal to the grating, in a plane at right angles to the slits. Then if $a$ is the width of the slits, and $b$ is the width of the opaque bars, the angle $\phi$ which expresses the difference in phase at $P$ of light from the two edges of a slit is

$$
\phi=\frac{2 \pi a \sin \theta}{\lambda}
$$

and the difference of phase of light from the two edges of a bar is

$$
\phi^{\prime}=\frac{2 \pi b \sin \theta}{\lambda} \text {. }
$$

The arc $c d$ is proportional to $a$, hence $\phi=k \frac{a}{\mathrm{R}}$, where $\mathrm{R}$ is the radius of the circle $c d e f$, and $k$ is a constant.

$$
\frac{2 \pi a \sin \theta}{\lambda}=k_{\mathrm{R}}^{a} \therefore \mathrm{R}=\frac{k \lambda}{2 \pi \sin \theta} \text {. }
$$

The length of the chord $c d$ expressing the amplitude due to a single slit may be represented by $l$, and we have

$$
l=2 \mathrm{R} \sin \frac{1}{2} \phi=k a \cdot\left(\frac{\sin \frac{\pi \alpha \sin \theta}{\lambda}}{\frac{\pi a \sin \theta}{\lambda}}\right) .
$$

Construct a circle, radius $r$ (in the above diagram smaller than $\mathrm{R}$ ), such that chords equal and parallel to chords $c d$, ef, \&c. will meet on its circumference.

The angle between the normals to consecutive chords is $\phi+\phi^{\prime}$, and since they meet on its circumference we have

$$
\frac{l}{2 r}=\sin \frac{1}{2}\left(\phi+\phi^{\prime}\right), \text { bence } r=\frac{l}{2 \sin \frac{1}{2}\left(\phi+\phi^{\prime}\right)} .
$$

But in the circle of radius $r$ the $n$ chords subtend an arc $n\left(\phi+\phi^{\prime}\right)$, and the resultant amplitude is therefore

$$
\mathrm{A}=2 r \sin \frac{n\left(\phi+\phi^{\prime}\right)}{2}=l \frac{\sin \frac{n\left(\phi+\phi^{\prime}\right)}{2}}{\sin \frac{\phi+\phi^{\prime}}{2}}
$$

and the intensity $I=A^{2}$. 
Substituting values of $\phi$ and $\phi^{\prime}$ we find

$$
\mathrm{A}=l \cdot \frac{\sin \left(\frac{n \pi(a+b) \sin \theta}{\lambda}\right)}{\sin \left(\frac{\pi(a+b) \sin \theta}{\lambda}\right)}
$$

the square of which is the usual expression for intensity.

The discussion of the maxima and minima becomes exceedingly simple from the point of view of the above construction.

It will be observed that as $\phi+\phi^{\prime}$ increases $r$ varies back and forth between the limits infinity and $l / 2$.

The principal mawima will be when the chords $l$ are all in the same phase, and hence form a straight line of length $n l$; this can only occur when the radius $r$ is infinitely great, that is when $\phi+\phi^{\prime}=2 \pi p$ or $(a+b) \sin \theta=p \lambda$ where $p$ is any whole number or zero. The corresponding intensities are $n^{2} l^{2}$.

After passing such a maximum, as $\phi+\phi^{\prime}$ increases $r$ becomes smaller until the curve subtended by the $n$ chords reaches ju-t completely around the circle. The intensity is then zero, and the are $n\left(\phi+\phi^{\prime}\right)$ equals $2 \pi n p+2 \pi$, or differs by $2 \pi$ from th at corresponding to the maximum.

As $\phi+\phi^{\prime}$ still farther increases $r$ continues to decrease till it reaches a value where the curve of chords reaches one and a half times around the circle, or where $n\left(\phi+\phi^{\prime}\right)=2 \pi n p+3 \pi$. This is very nearly a subordinate maximum, though not exactly, because, since $r$ is decreasing, the maximum value will be the chord of an arc a little less than $3 \pi$ for which the radius is greater. It is easily found by differentiation in the usual way.

Taking the approximate value $n\left(\phi+\phi^{\prime}\right)=3 \pi$, the amplitude being $2 r$ becomes

$$
\frac{l}{\sin \left(\frac{3 \pi}{2 n}\right)}
$$

and as $n$ is large we have

$$
\mathrm{A}=\frac{2 n l}{3 \pi} \text { or } \mathrm{I}=n^{2} l^{2}\left(\frac{4}{9 \pi^{2}}\right),
$$

that is, the first subordinate maximum has about $\frac{1}{2} \cdot \frac{2}{2}$ the intensity of the principal maximum.

As $r$ further decreases there will be a subordinate maximum every time the curve of the $n$ chords reaches around the circle a whole number of times plus an additional half turn. Between two consecutive principal maxima there will thus be a series of $n-2$ subordinate maxima with absolute minima 
between them, and the intensity of the smallest of these maxima will be $l^{2}$.

The dependence of the width of the lines in the diffractionspectrum on the effective width of the grating is easily shown as follows :- Suppose it is assumed that the first maximum practically fades out of sight when $n\left(\phi+\phi^{\prime}\right)$ differs from $2 \pi n p$, its value at the principal maxima, by $\pm \frac{3 \pi}{2}$, in which case the intensity is less than one-tenth that of the maximum Then,

and

$$
\begin{gathered}
\frac{n \pi(a+b) \sin \theta}{\lambda}=2 \pi n p \pm \frac{3 \pi}{2}, \\
\sin \theta=\frac{2 \lambda p}{a+b} \pm \frac{3 \lambda}{2 n(a+b)} \\
d \theta=\frac{d(\sin \theta)}{\cos \theta}=\frac{3 \lambda}{2 n(a+b) \cos \theta}=\frac{3 \lambda}{2 w \cos \theta} .
\end{gathered}
$$

where $u$ represents the width of the grating.

The angular width of a line in the spectrum is $2 d \theta$ or

$$
\alpha=\frac{3 \lambda}{w \cos \theta}
$$

assuming that the line fades out where the intensity is onetenth of the maximum.

In ruled gratings, whether transmitting or reflecting, the value of $l$ depends on the nature of the grooves cut by the ruling diamond as well as on the angle $\theta$, and therefore the expression given above for $l$, which assnmes a simple slit, does not apply. But the summation holds good in any case if the ruling is uniform.

Amherst College, U.S.A.

\section{Measurements of Small Resistances. By Albert Campbell, B.A.*}

$7 \mathrm{VE}$ object of this paper is to give a brief account of a number of measurements of a set of low-resistance standards belonging to the National Physical Laboratory; these tests were made partly with a view to comparing various methods of measurement, and it is from this point of view that they derive their main interest. The resistances were of the oil-cooled type and had separate potential terminals. They were made of manganin, and their nominal values were

* Communicated by the Physical Society : read March 13, 1903. Plil. Mag. S. 6. Vol. 6. No. 31. July 1903. 